Supporting information

\title{
Cancer Cytomembrane-Cloaked Prussian Blue Nanoparticles Enhance the Efficacy of Mild- Temperature Photothermal Therapy by Disrupting Mitochondrial Functions of Cancer Cells
}

Pei Wang a, b, Ranjith Kumar Kankala ${ }^{\mathrm{a}}$, Biaoqi Chen ${ }^{\mathrm{a}}$, Yang Zhang ${ }^{\mathrm{c}}$, Mingzhi Zhu ${ }^{\mathrm{a}}$, Xuemei Li ${ }^{\mathrm{a}}$, Ruimin Long ${ }^{\mathrm{a}}$, Dayun Yang ${ }^{\mathrm{d}}$, Rumen Krastev ${ }^{\mathrm{e}}$, Shibin Wang ${ }^{\mathrm{a}}$, Xin Xiong ${ }^{\mathrm{f}, *}$, Yuangang Liu ${ }^{\mathrm{a}, *}$

${ }^{a}$ Fujian Provincial Key Laboratory of Biochemical Technology, Institute of Pharmaceutical Engineering, Huaqiao University, Xiamen 361021, P. R. China

${ }^{b}$ Jiangxi Key Laboratory of Stomatology and Biomedicine, School of Stomatology, Nanchang University, Nanchang 330006, P. R. China

'State Key Laboratory of Molecular Vaccinology and Molecular Diagnostics, Center for Molecular Imaging and Translational Medicine, School of Public Health, Xiamen University, Xiamen 361021, P. R. China

${ }^{d}$ Institute for Translational Medicine, School of Basic Medical Science, Fujian Medical University, Fuzhou 350122, P. R. China

${ }^{e}$ Faculty for Applied Chemistry, Reutlingen University, Alteburgstr, 150, 72762 Reutlingen, Germany

${ }^{f}$ NMI Natural and Medical Sciences Institute, University of Tübingen, Markwiesenstr, 55, 72770 Reutlingen, Germany

*Corresponding authors:

ygliu@hqu.edu.cn (Y. Liu); Xin.xiong@nmi.de (X. Xiong) 

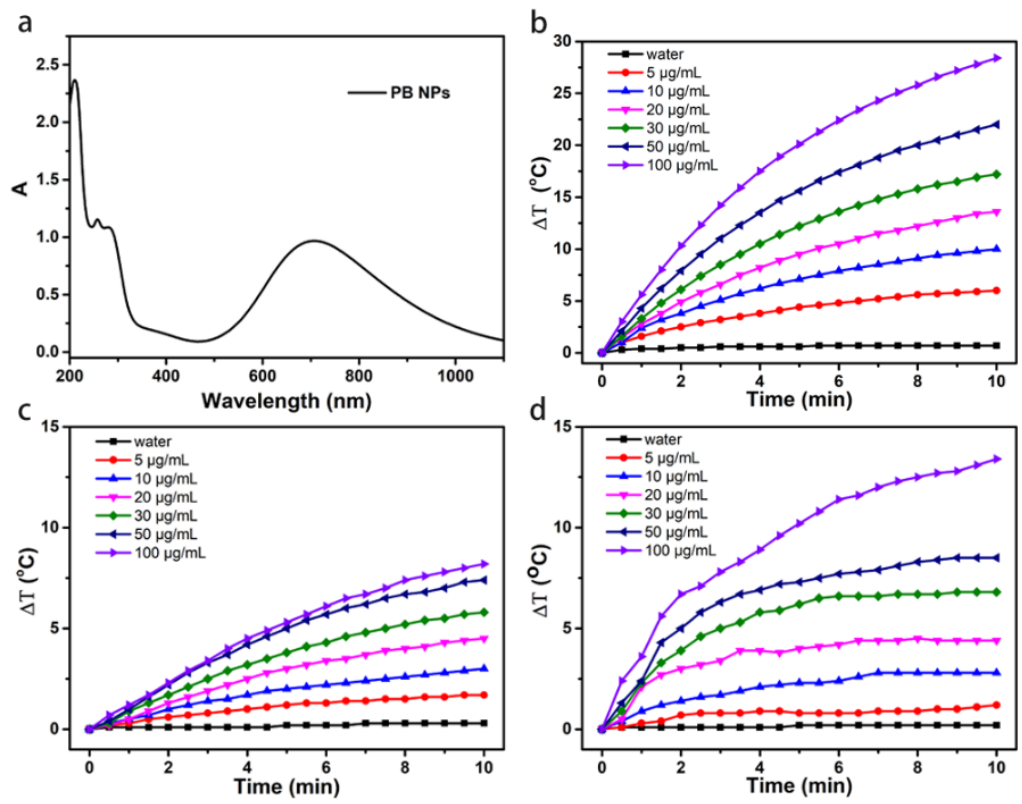

Figure S1. (a) UV-Vis-NIR absorption spectrum of PB NPs. The temperature changes of aqueous dispersions of PB NPs with the various concentrations under the irradiation of 808-nm laser for $10 \mathrm{~min}$ (b) at $1 \mathrm{~cm}$ spot diameter and $1 \mathrm{~mL}$ solution, (c) at $0.5 \mathrm{~cm}$ spot diameter and $1 \mathrm{~mL}$ solution, and $(d)$ at $0.5 \mathrm{~cm}$ spot diameter and $0.1 \mathrm{~mL}$ solution.
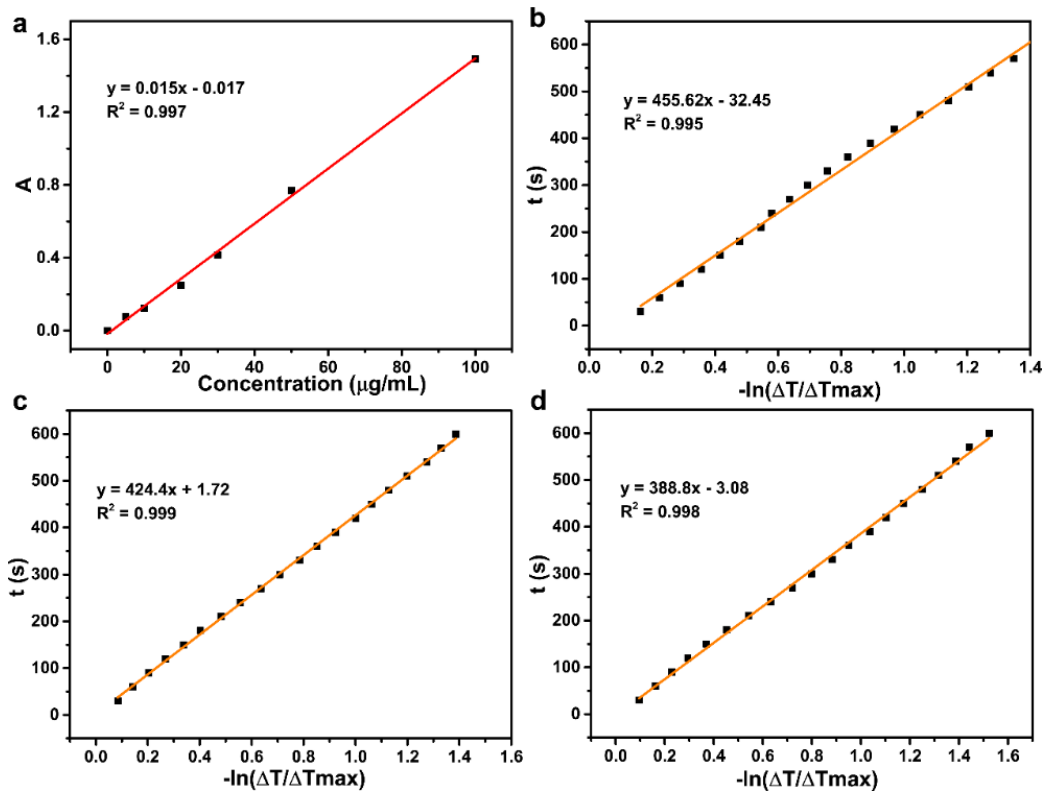

Figure S2. (a) Calibration curves of $P B N P s$ at the wavelength of $808 \mathrm{~nm}$. The fitting line of PB NPs cooling at (b) 10, (c) 20, and (d) $50 \mu \mathrm{g} / \mathrm{mL}$. 

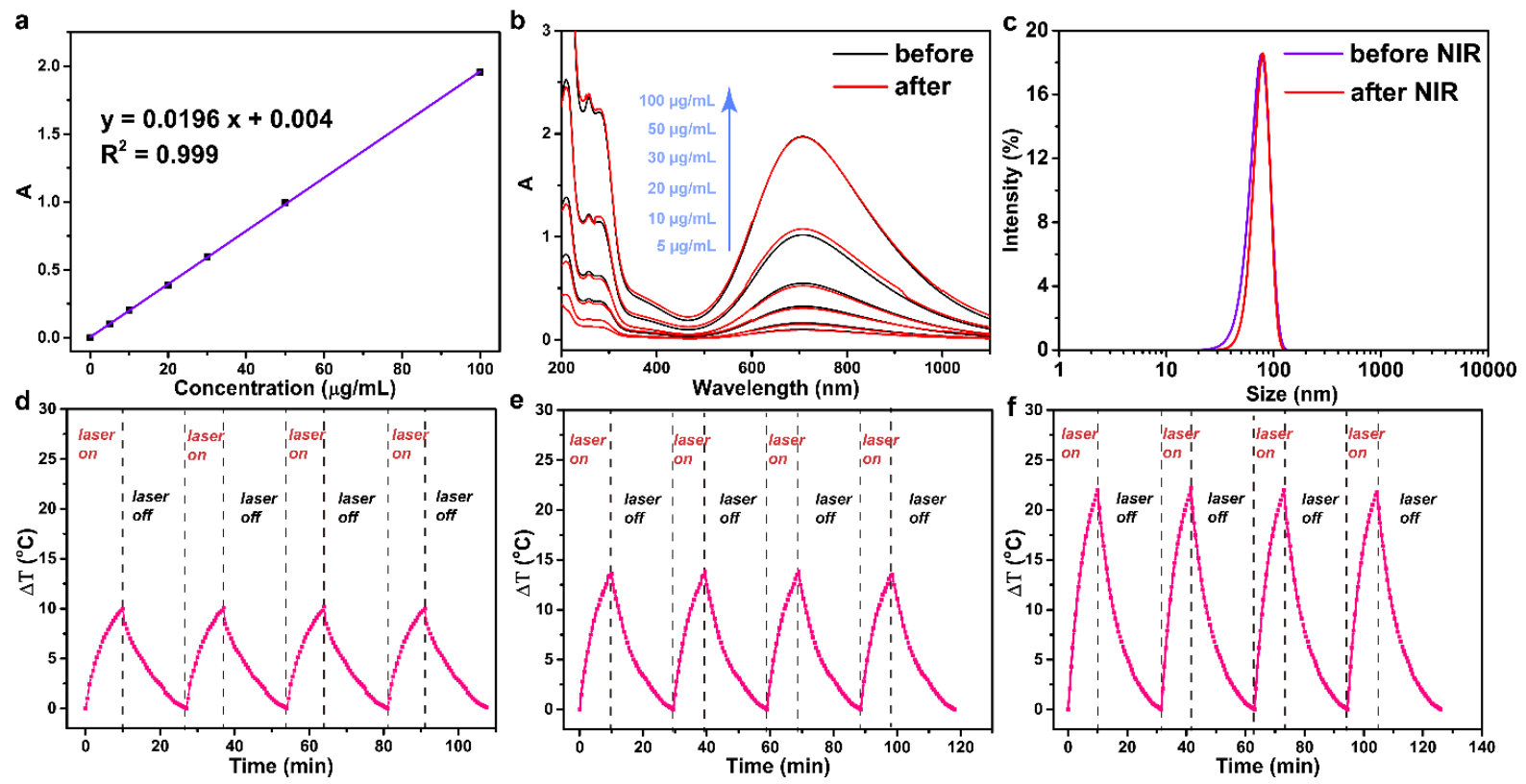

Figure S3. (a) Calibration curve of $P B N P$ s at the wavelength of $700 \mathrm{~nm}$. (b) UV-Vis-NIR absorption spectrum and (c) hydration size of $P B N P$ s before and after irradiation. Temperature variations of PB NPs at the concentration of (d) $10 \mu \mathrm{g} / \mathrm{mL}$, (e) $20 \mu \mathrm{g} / \mathrm{mL}$, and (f) $50 \mu \mathrm{g} / \mathrm{mL}$ under the continuous irradiation of an 808-nm laser for 4 cycles at $1 \mathrm{~W} \mathrm{cm-}^{2}$.
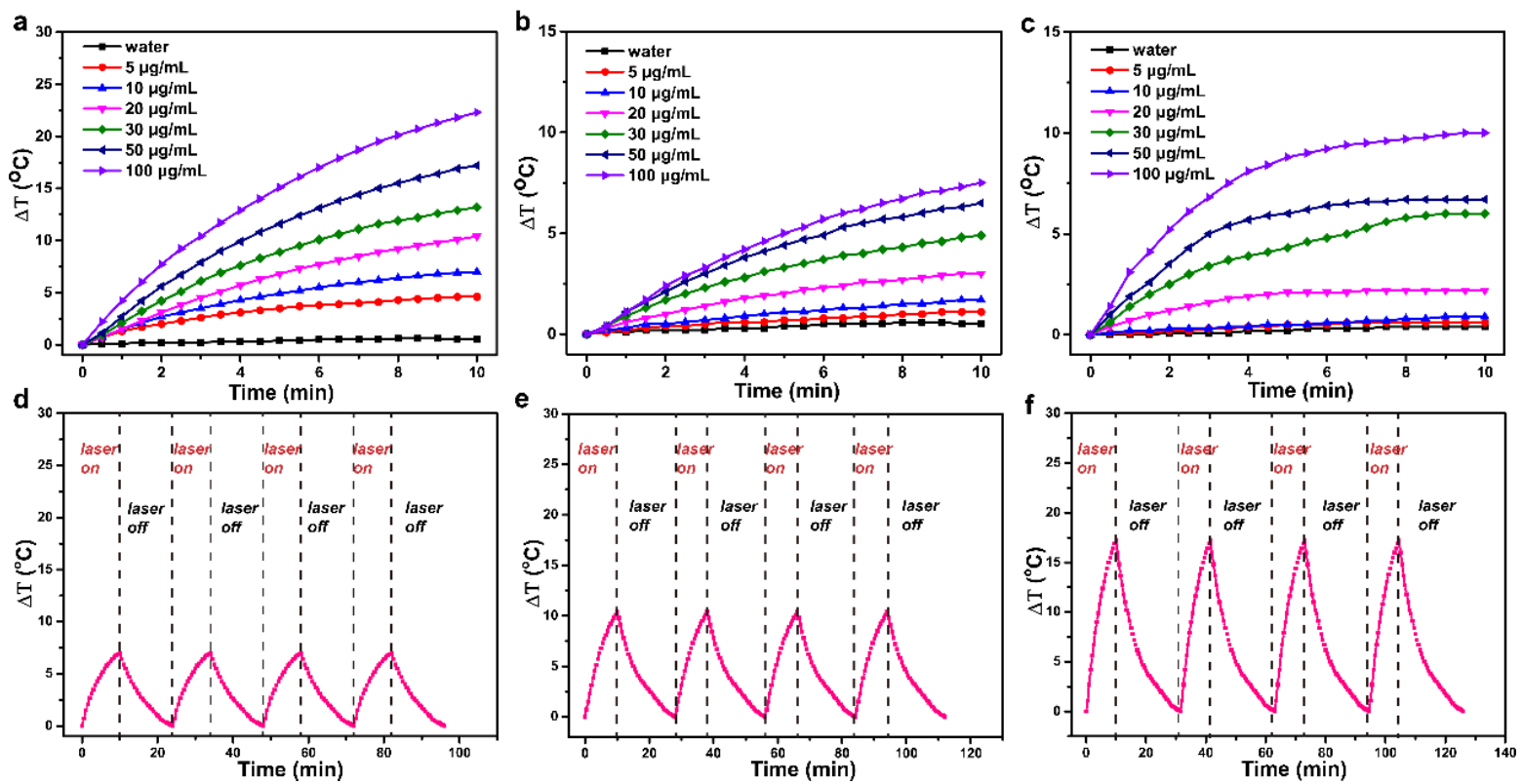

Figure S4. Temperature changes of aqueous dispersions of $P G$ NPs with the various concentrations under the irradiation of an 808-nm laser for $10 \mathrm{~min}$ (a) at $1 \mathrm{~cm}$ spot diameter and 
$1 \mathrm{~mL}$ solution, (b) at $0.5 \mathrm{~cm}$ spot diameter and $1 \mathrm{~mL}$ solution, and (c) at $0.5 \mathrm{~cm}$ spot diameter and $0.1 \mathrm{~mL}$ solution. Temperature variations of PG NPs at the concentration of (d) $10 \mu \mathrm{g} / \mathrm{mL}$, (e) 20 $\mu \mathrm{g} / \mathrm{mL}$, and $(f) 50 \mu \mathrm{g} / \mathrm{mL}$ under the continuous irradiation of an 808-nm laser for 4 cycles at $1 \mathrm{~W}$ $\mathrm{cm}^{2}$.
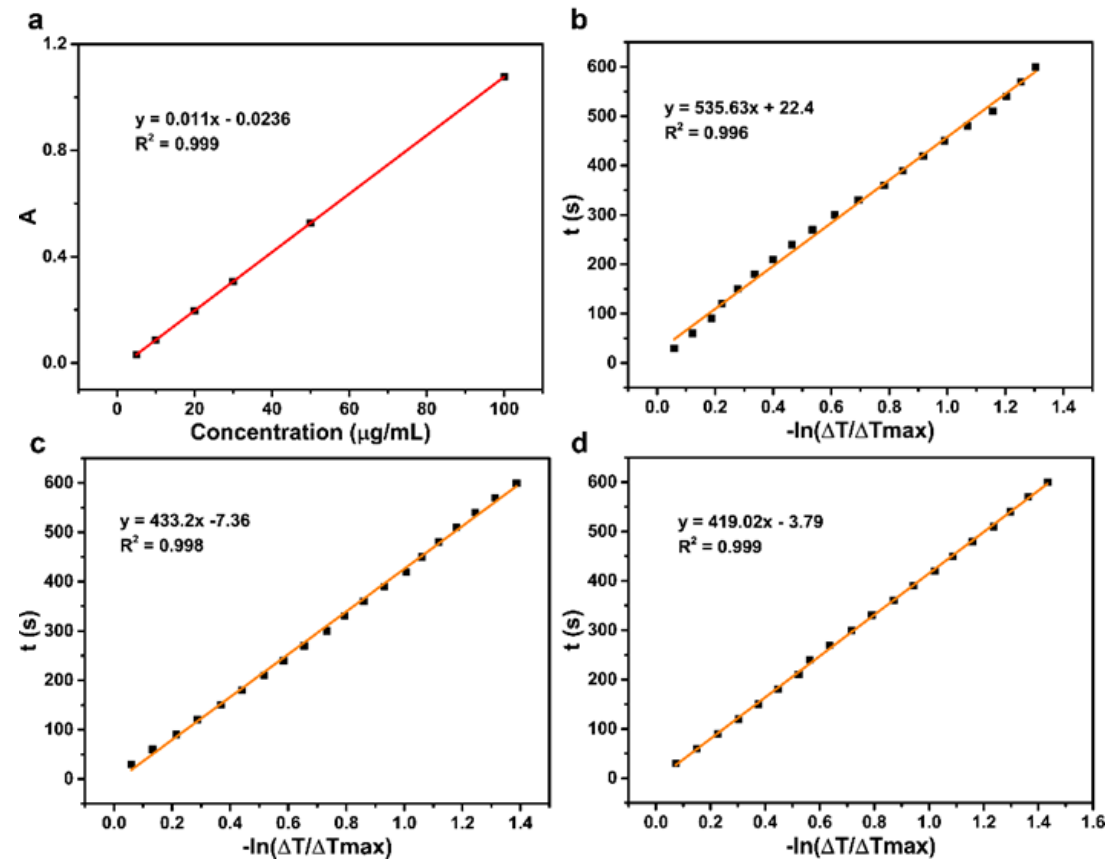

Figure S5. (a) Calibration curves of PG NPs at the wavelength of $808 \mathrm{~nm}$. The fitting line of PG NPs cooling at (b) 10, (c) 20, and (d) $50 \mu \mathrm{g} / \mathrm{mL}$.
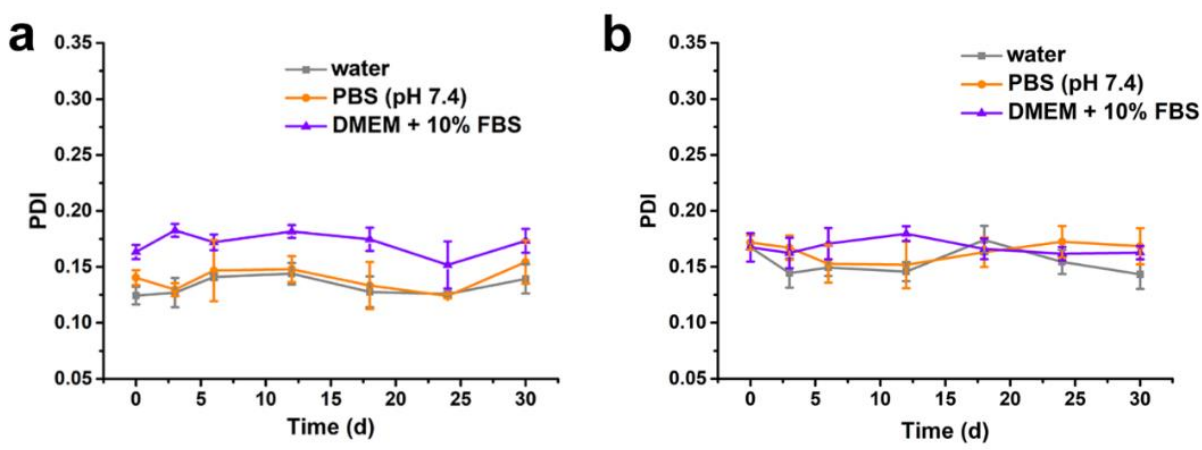

Figure S6. Polydispersity of (a) PB NPs and (b) HmPG NPs in water, PBS (pH 7.4) and DMEM medium for 30 days. 


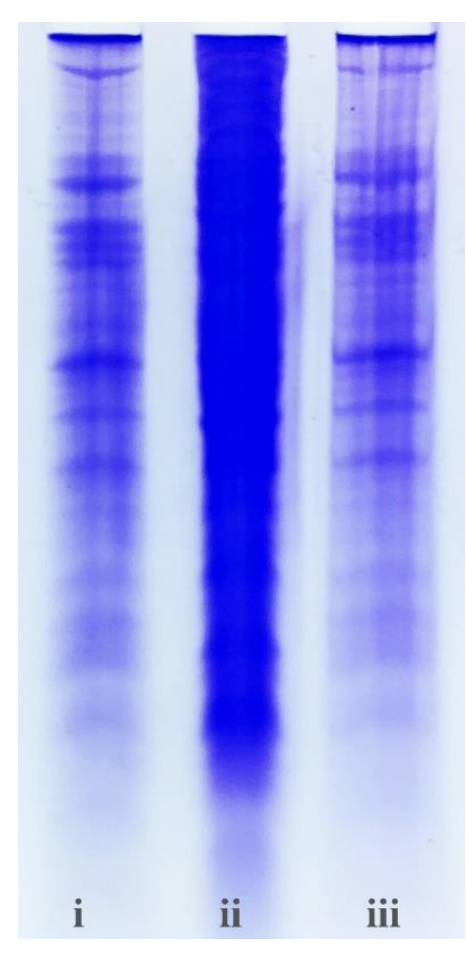

Figure S7. SDS-PAGE gel electrophoresis of i) cell membrane protein, ii) cell cytoplasmic protein, and iii) HmPG NPs.
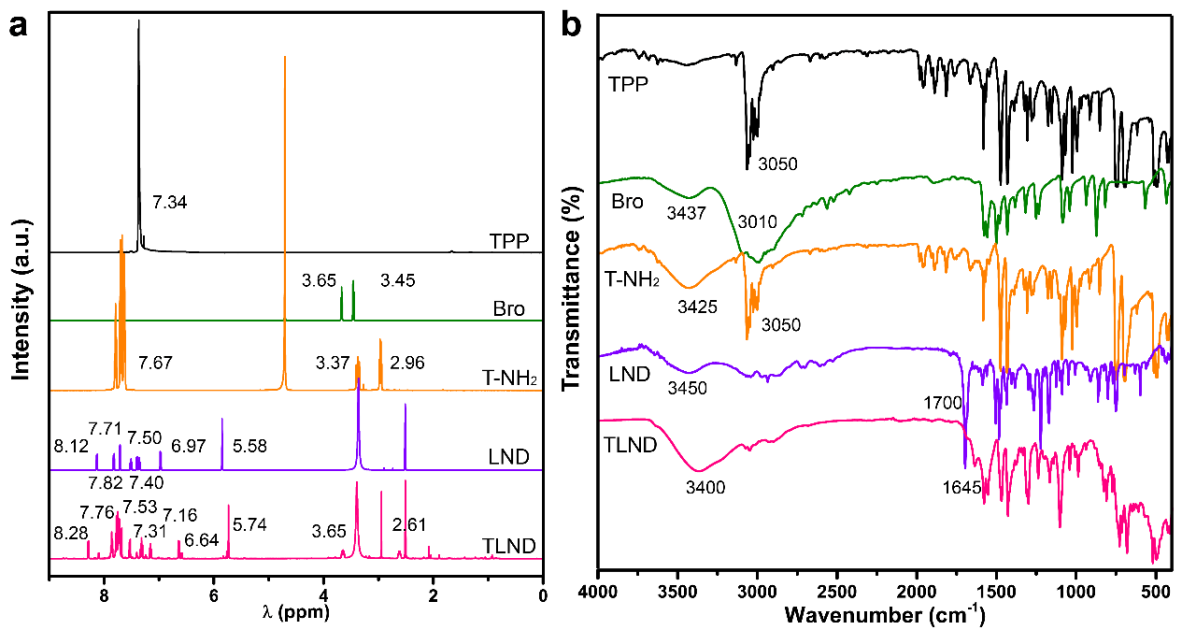

Figure S8. (a) ${ }^{1} H$ NMR spectra, (b) FTIR spectra of TPP, Bro, T-NH, LND, and TLND. 


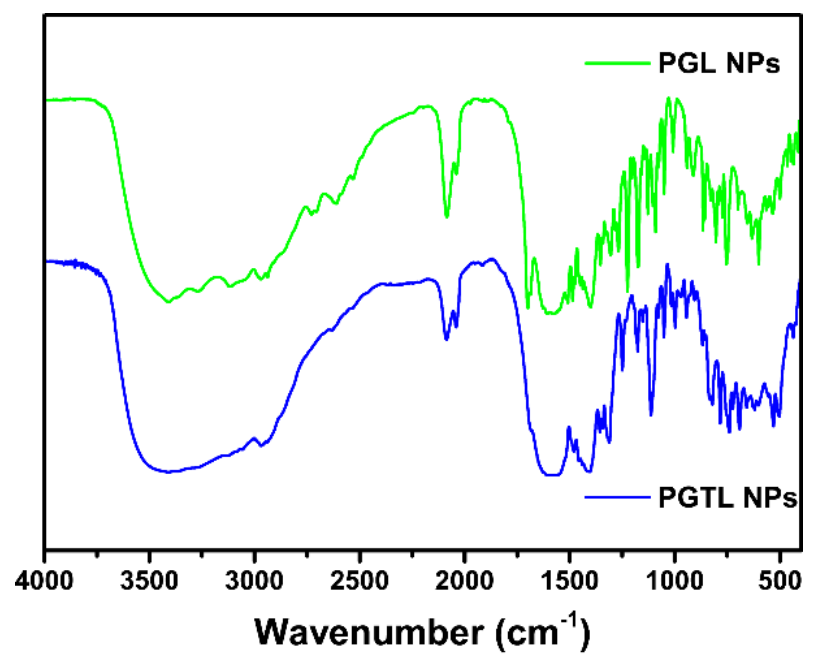

Figure S9. FTIR spectra of HmPGL and HmPGTL NPS.
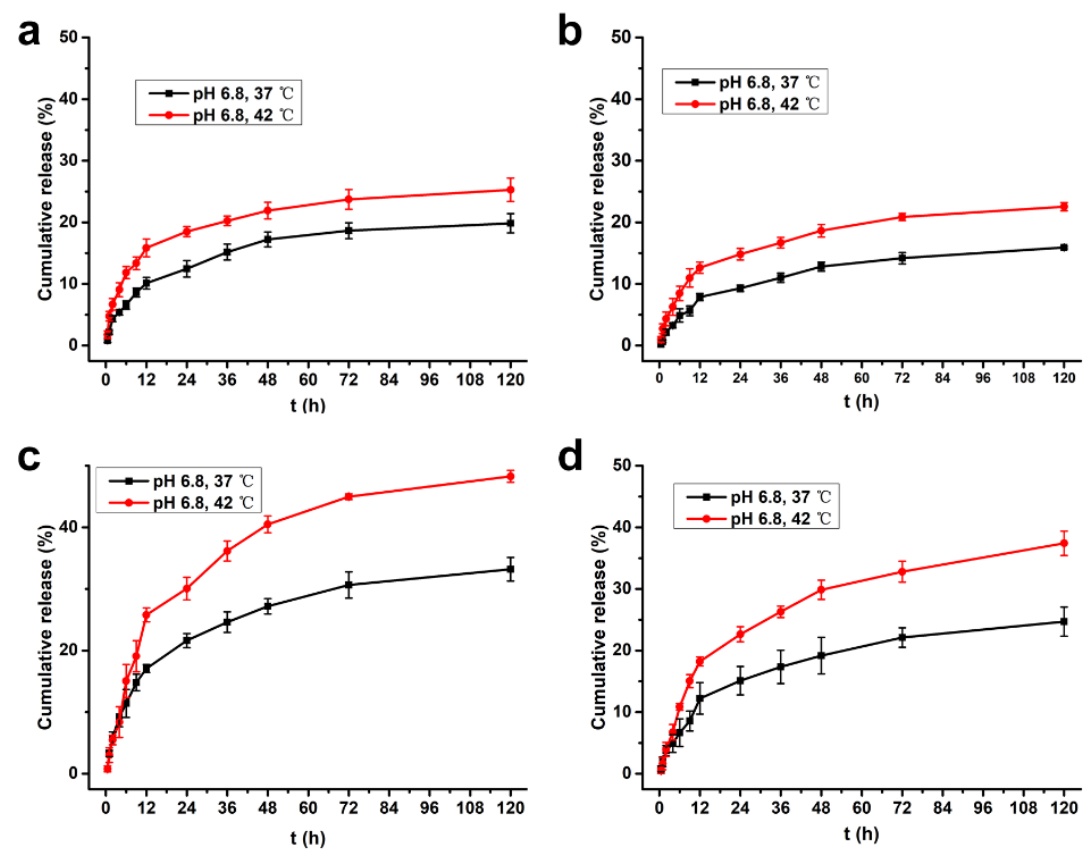

Figure S10. Cumulative release profiles of $L N D$ and TLND from (a) PGL NPS, (b) HmPGL NPS, (c) PGTL NPs, and (d) HmPGTL NPs in PBS (pH 6.8) at temperature 42 or $37^{\circ} \mathrm{C}$. 


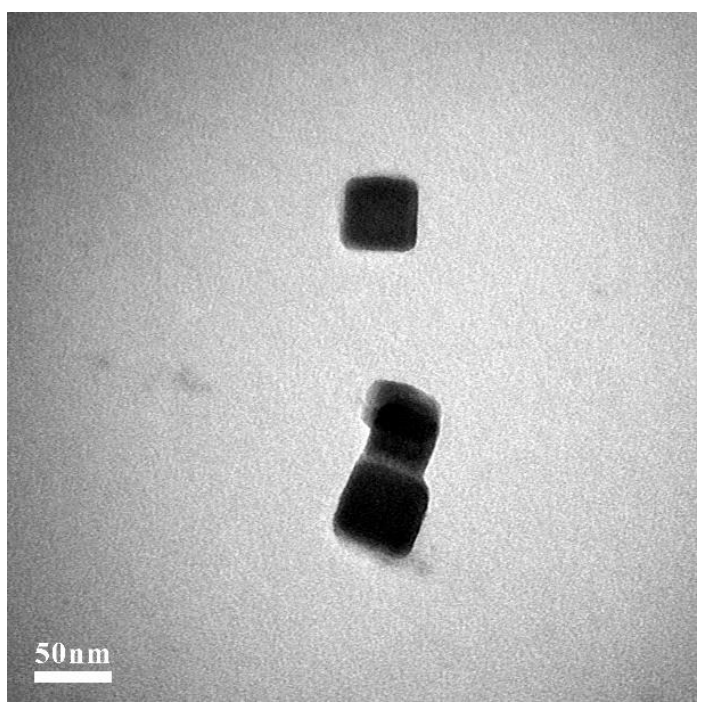

Figure S11. TEM image of PG NPS after incubation in PBS (pH 5.0) for $4 \mathrm{~h}$.
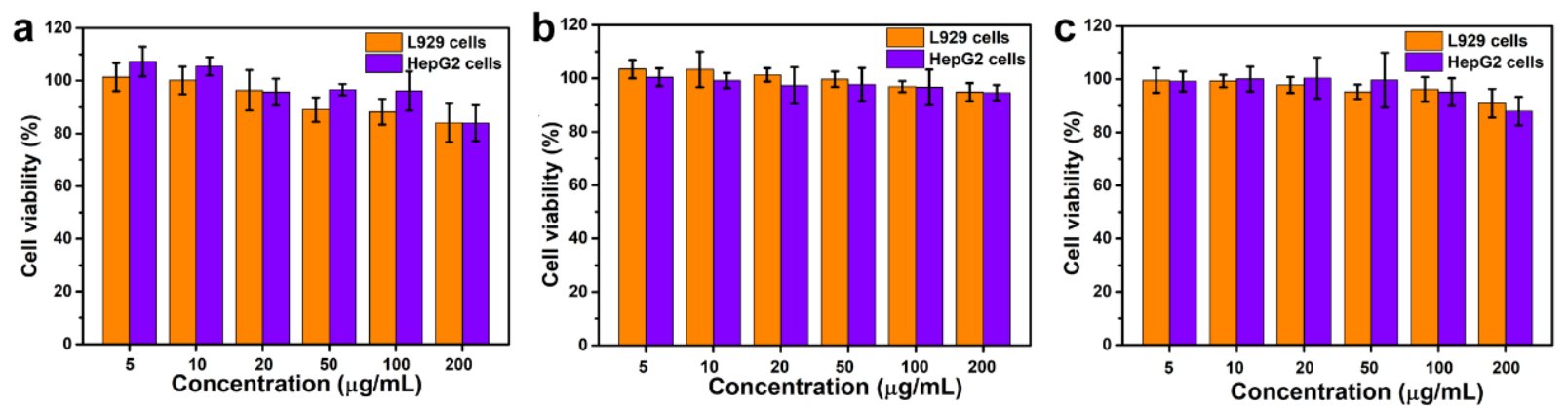

Figure S12. Relative cell viability of L929 and HepG2 cells after incubation with (a) PB NPs, (b) $P G N P s$, and (c) HmPG NPs at various concentrations for $48 \mathrm{~h}$. 

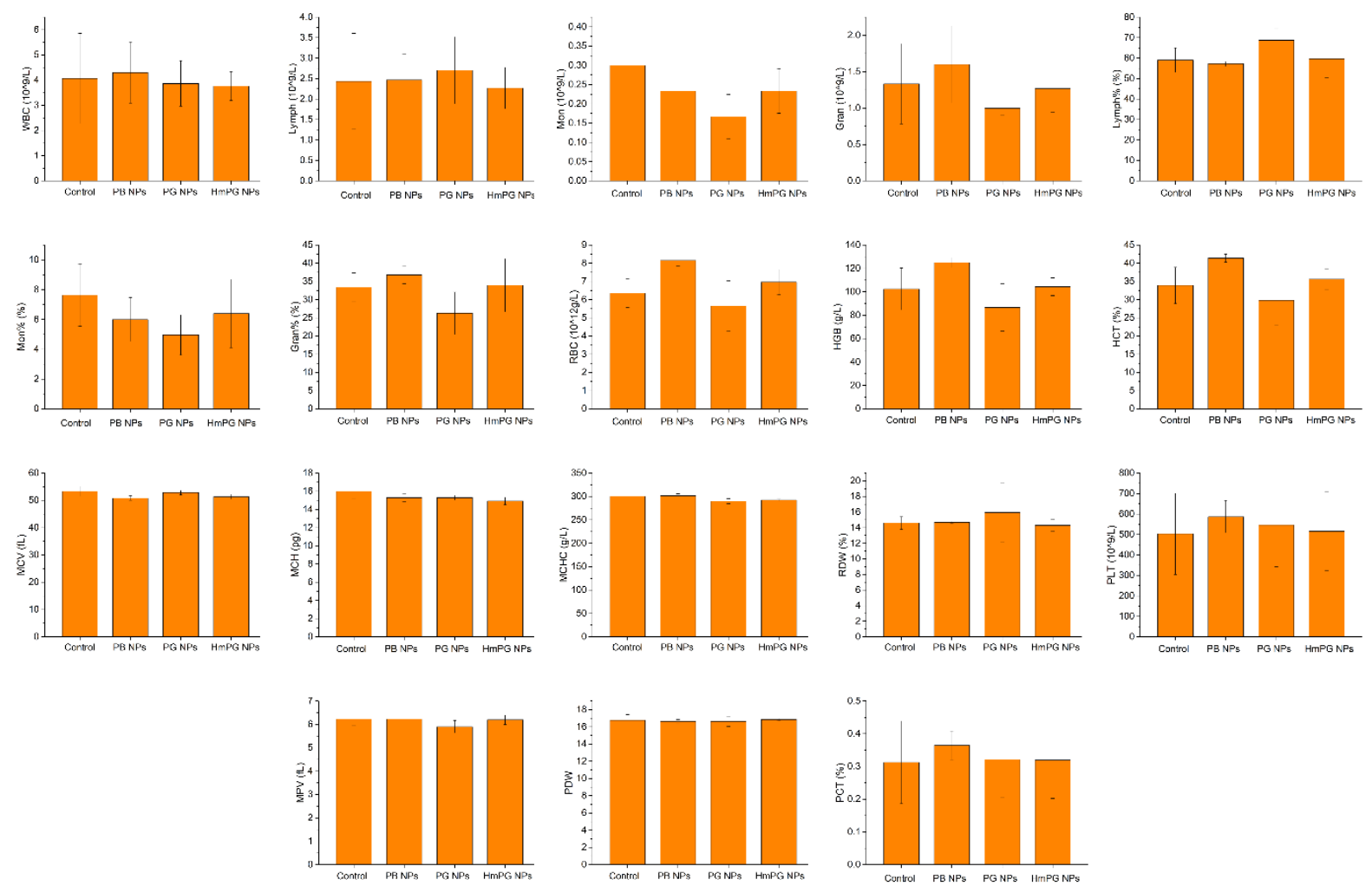

Figure S13. Hematological assay. The related indexes of the mice after $7 d$ injection.

a

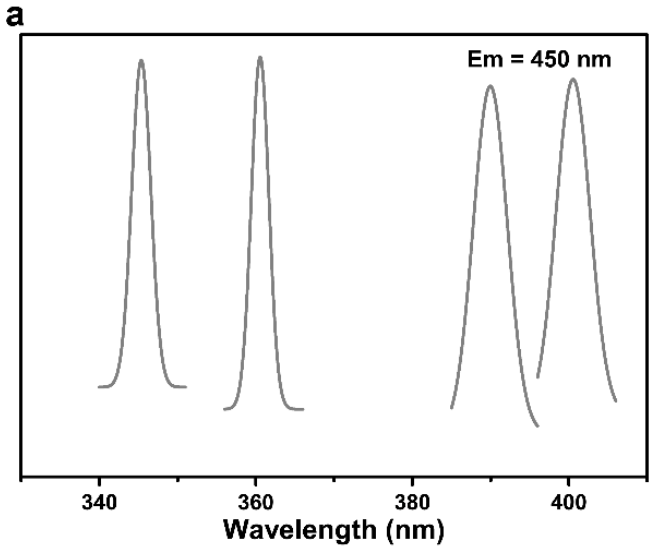

b

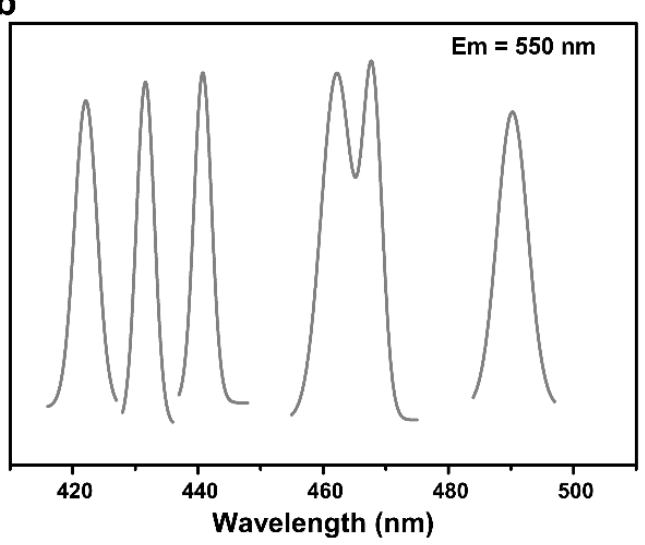

Figure S14. Excitation spectra of PB NPs under the emission wavelength of (a) $450 \mathrm{~nm}$ and (b) $550 \mathrm{~nm}$. 

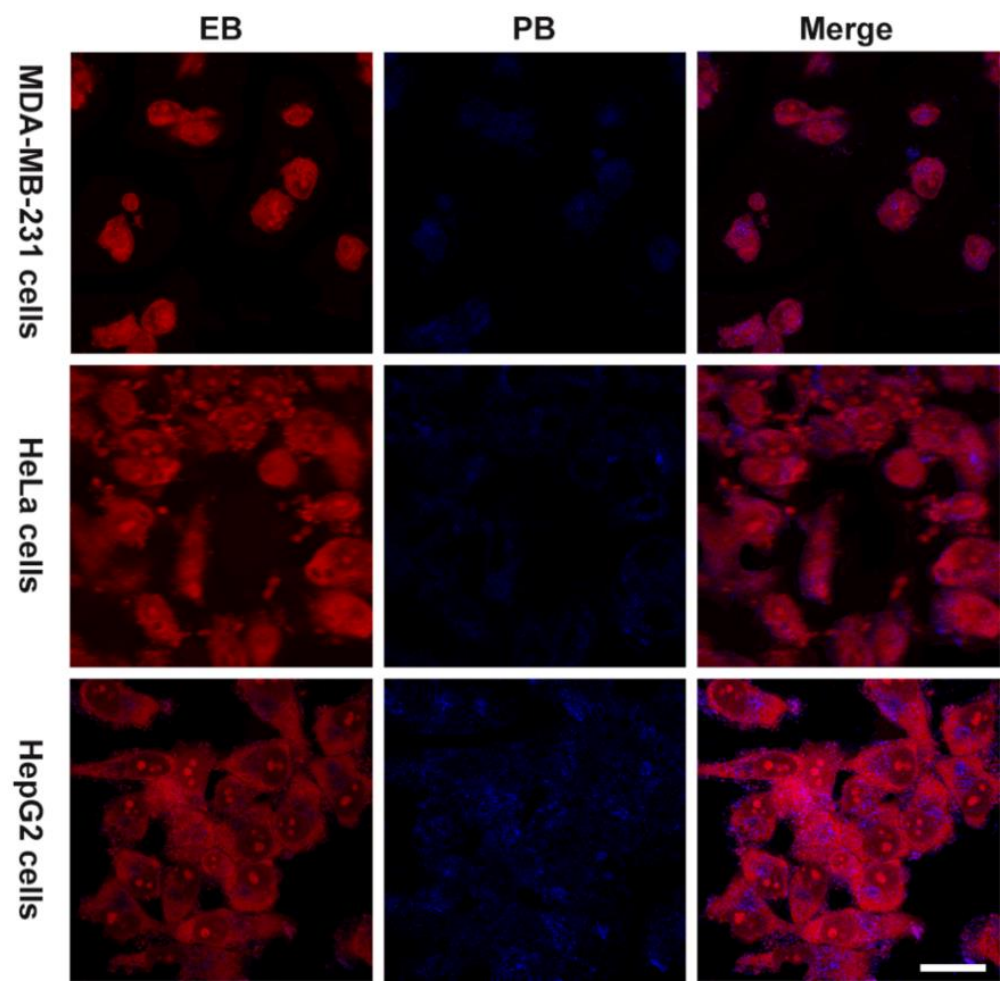

Figure S15. CLSM images showing the cellular internalization of HmPG NPs $($ scale bar $=25 \mu \mathrm{m})$.
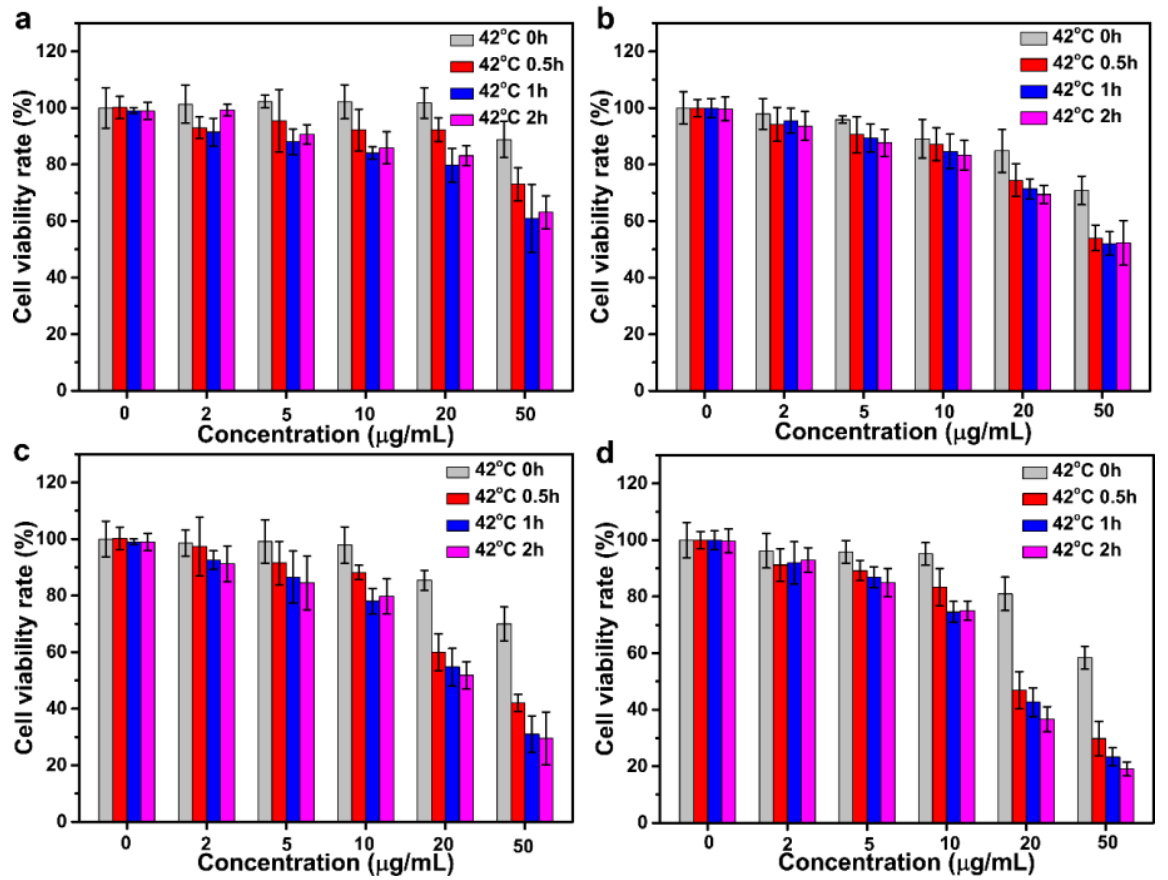

Figure S16. Relative viability of HepG2 cells after incubation with LND for a total incubation time 
of (a) 24 and (b) $48 \mathrm{~h}$ and TLND for a total incubation time of (c) 24 and (d) $48 \mathrm{~h}$ at $42{ }^{\circ} \mathrm{C}$ for various periods and then cultured at $37^{\circ} \mathrm{C}$.
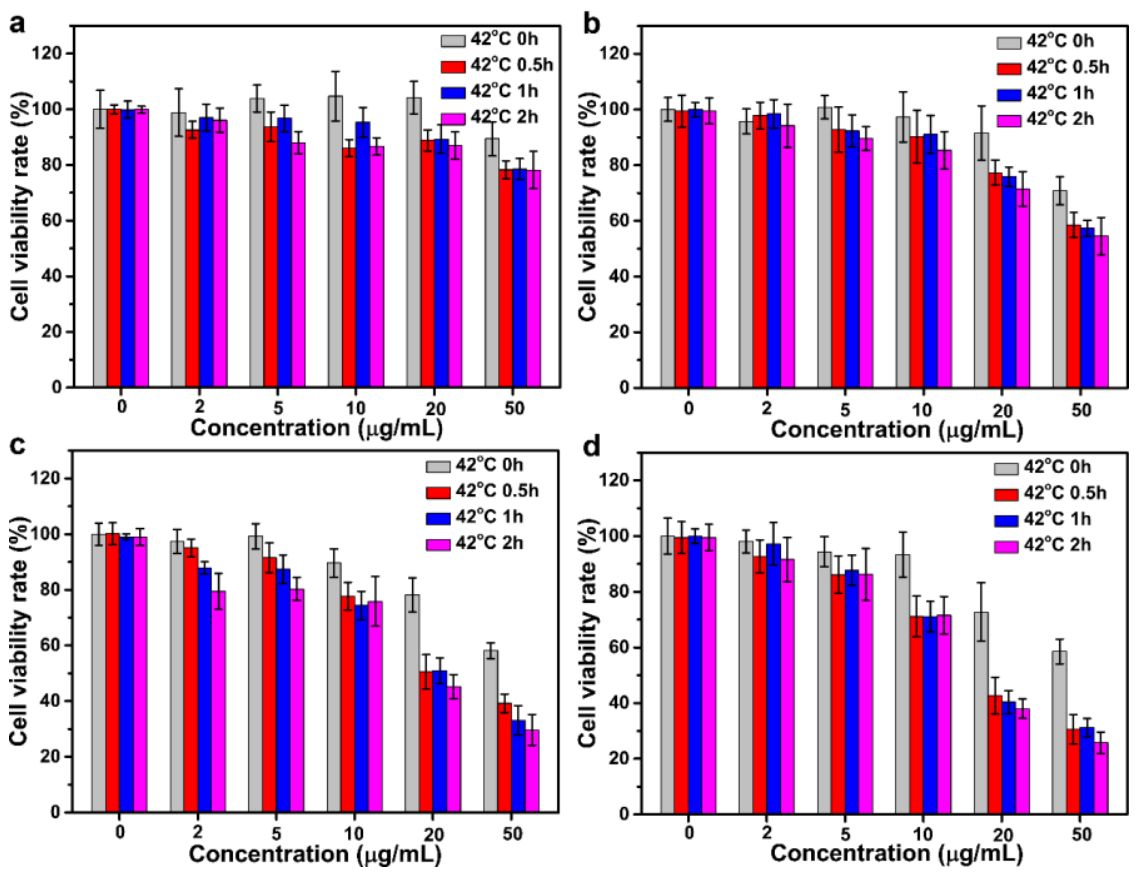

Figure S17. Relative viability of HepG2 cells after incubation with HmPGL NPs for a total incubation time of (a) 24 and (b) $48 \mathrm{~h}$ and HmPGTL NPs for a total incubation time of (c) 24 and (d) 48 h at $42^{\circ} \mathrm{C}$ for various periods and then cultured at $37^{\circ} \mathrm{C}$ (in terms of drug concentration of $20 \mu \mathrm{g} / \mathrm{mL}$ ).

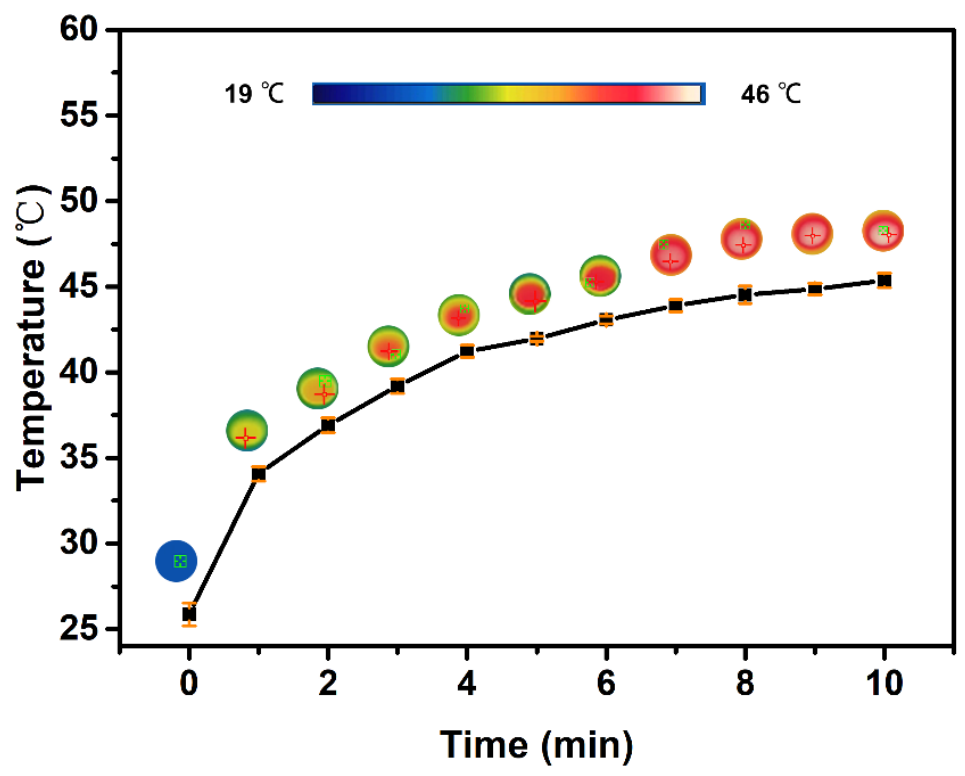


Figure S18. IR thermographic images of HepG2 cells and the in vitro temperature variations under the irradiation of $808 \mathrm{~nm}$ laser for $10 \mathrm{~min}$ at a power density of $1 \mathrm{~W} \mathrm{cm-}^{2}$ in 96-cell plate.

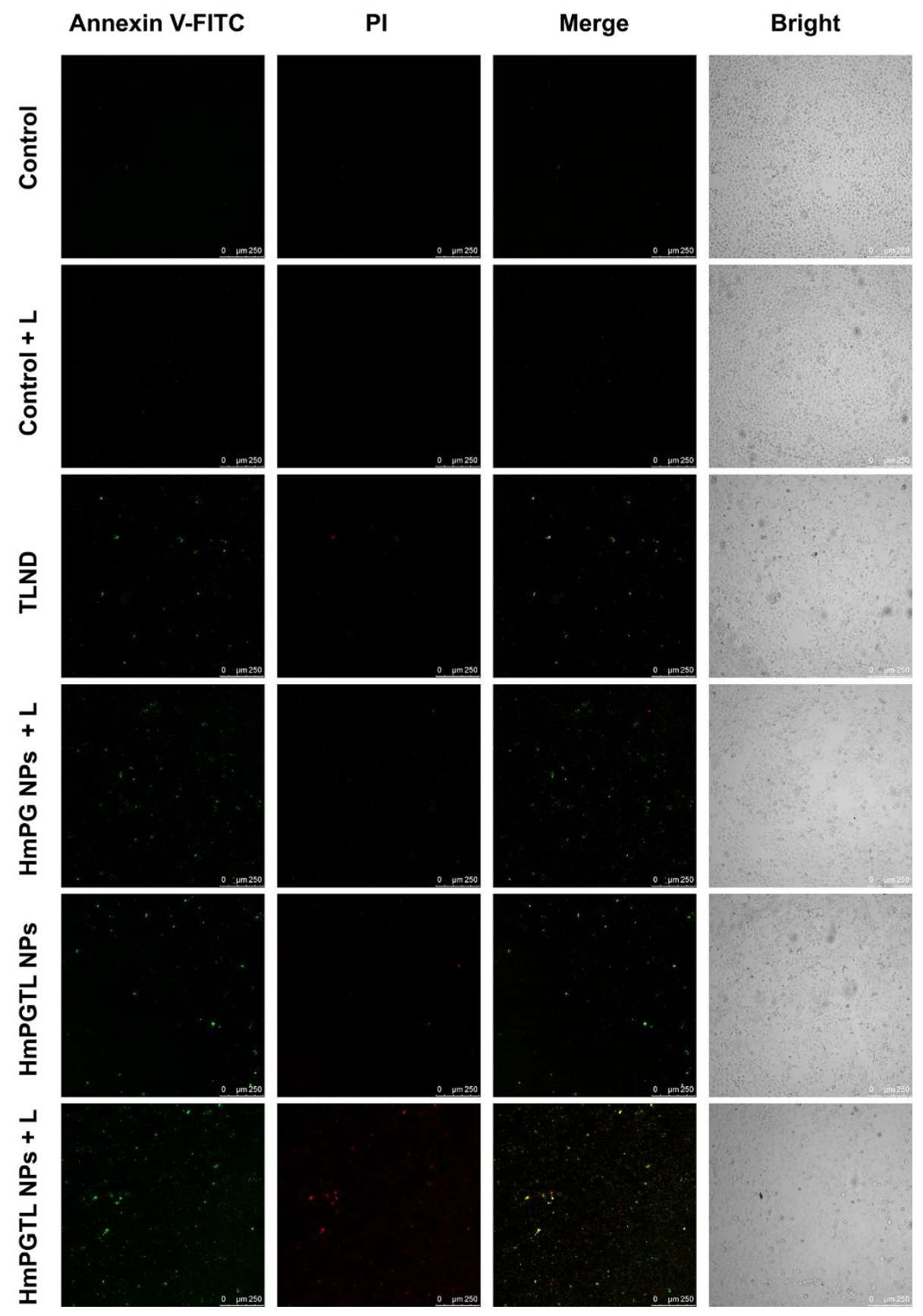

Figure S19. Apoptosis determination via membrane permeability. CLSM images showing the HepG2 cells stained using Annexin V-FITC/PI after treatment with TLND and HmPGTL NPS, compared with negative control $(100 \times)$. 

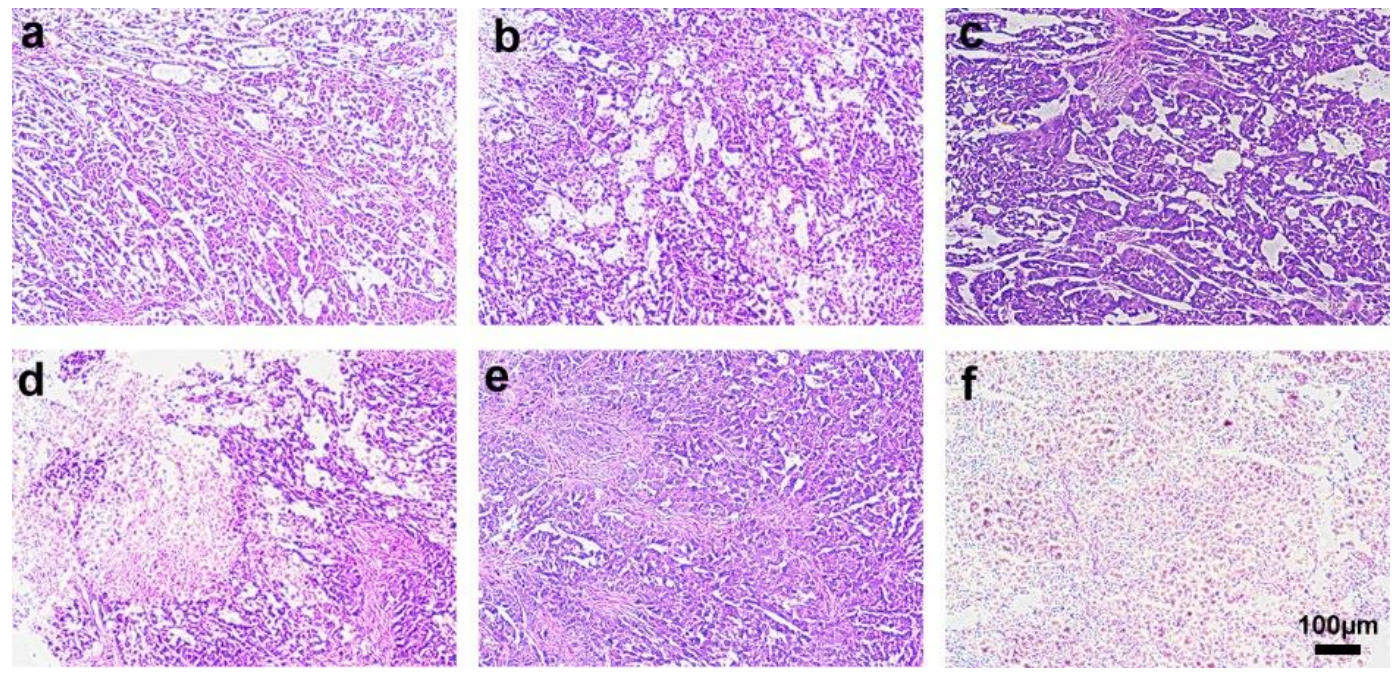

Figure S20. Representative images of tumor tissue after treatment with (a) $P B S,(b) P B S+L,(c)$ HmPG NPs, (d) HmPG + L, (e) HmPGTL NPs, and (f) HmPGTL NPs + L, in HepG2 tumor bearing nude mice at day 4. (Scale bar $=100 \mu \mathrm{m})$.

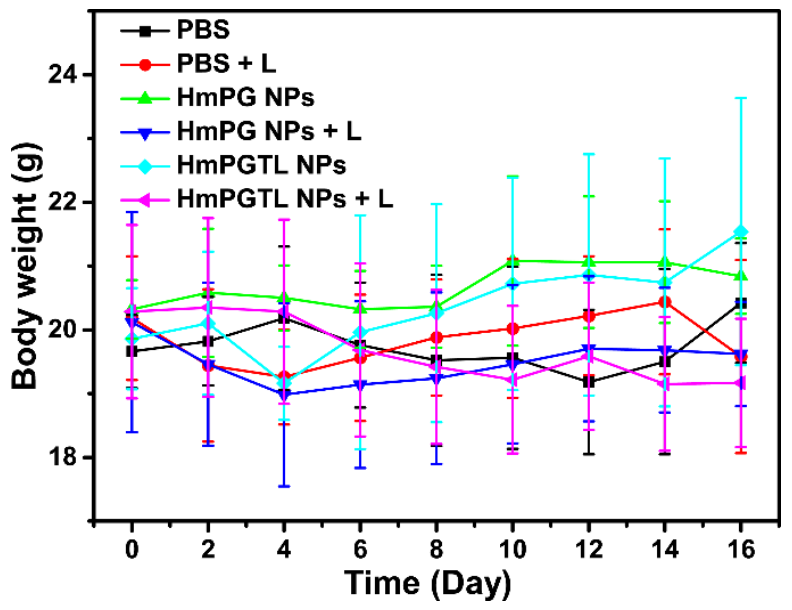

Figure S21. The body weights of HepG2 tumor-bearing nude mice measured every other day in different treatment groups. 


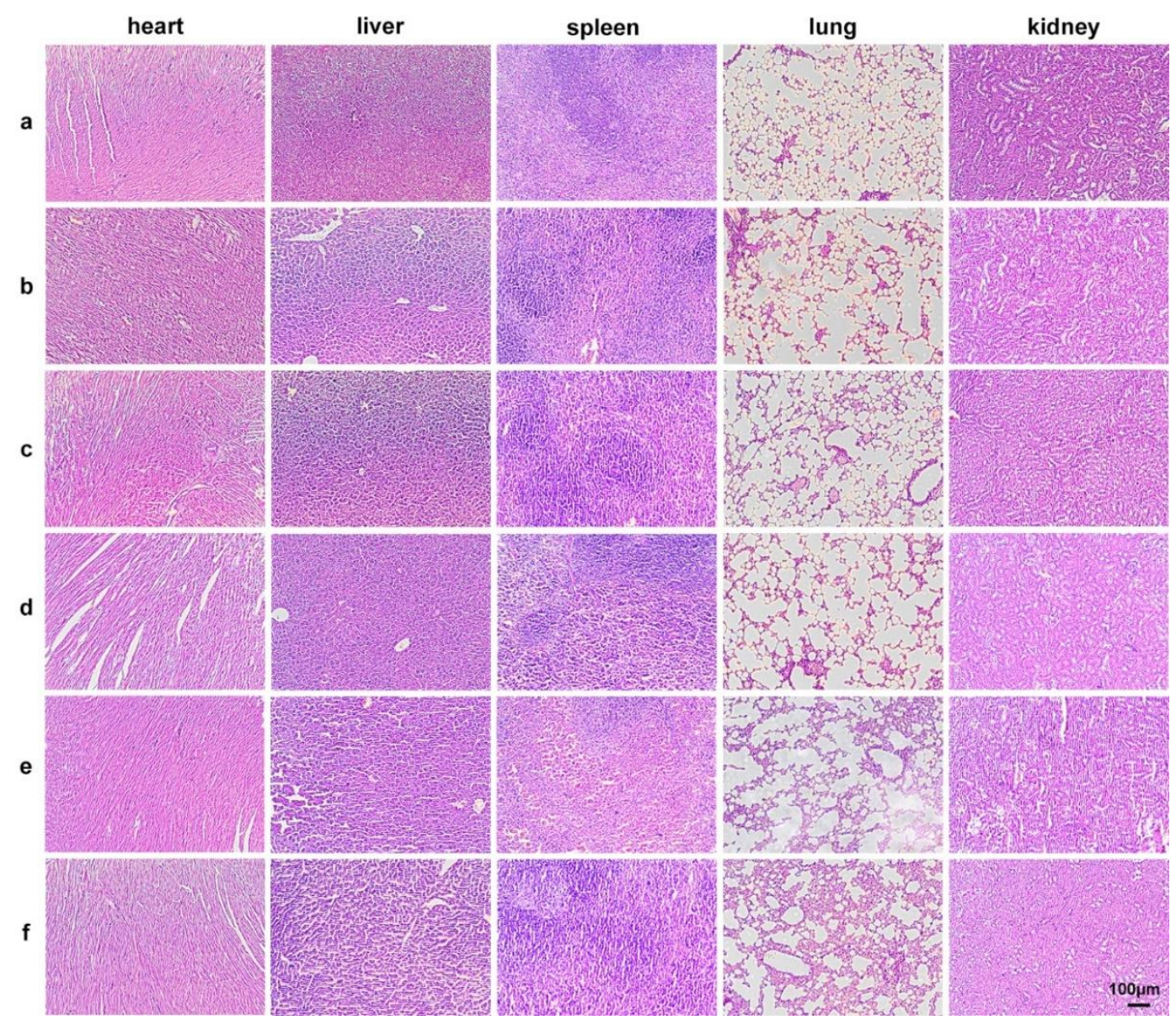

Figure S22. Representative images of major mouse organs (heart, liver, spleen, lung, and kidney) after treatment with (a) PBS, (b) PBS + L, (c) HmPG NPs, (d) HmPG NPs + L, (e) HmPGTL NPs, and $(f)$ HmPGTL NPs + L, treated HepG2 tumor-bearing nude mice at day 16. (Scale bar = 100 $\mu m)$. 
Table S1. The experimental results showing the changes in body weight of mice after intravenously injected with PBS, PB NPS, PG NPs, and HmPG NPs for 7 days.

\begin{tabular}{|c|c|c|c|c|c|c|c|c|c|c|c|c|}
\hline \multirow{2}{*}{ Time } & \multirow{2}{*}{ Sample } & \multicolumn{11}{|c|}{ Body weight (g) } \\
\hline & & \multicolumn{5}{|c|}{ male } & \multicolumn{5}{|c|}{ female } & \multirow{2}{*}{$\frac{\text { Average }}{30.7 \pm 2.1}$} \\
\hline & $a$ & 31.9 & 33.4 & 30.9 & 32.2 & 33.3 & 31.0 & 26.8 & 30.1 & 29.1 & 28.6 & \\
\hline 0 & $b$ & 37.3 & 33.2 & 36.0 & 31.3 & 34.0 & 29.1 & 28.4 & 30.0 & 32.0 & 30.3 & $32.2 \pm 2.9$ \\
\hline \multirow[t]{3}{*}{ day } & C & 32.1 & 31.4 & 31.9 & 31.1 & 30.0 & 29.3 & 29.3 & 29.5 & 30.8 & 29.3 & $30.5 \pm 1.1$ \\
\hline & $d$ & 31.2 & 31.8 & 31.2 & 31.7 & 30.0 & 27.8 & 28.2 & 30.0 & 28.1 & 27.9 & $29.8 \pm 1.7$ \\
\hline & a & 34.4 & 33.1 & 34.2 & 34.2 & 36.1 & 29.5 & 32.5 & 29.2 & 30.1 & 29.8 & $32.3 \pm 2.5$ \\
\hline 1 & $b$ & 33.1 & 38.1 & 32.2 & 34.0 & 34.6 & 30.2 & 30.6 & 29.4 & 29.5 & 27.9 & $32.0 \pm 3.1$ \\
\hline \multirow[t]{3}{*}{ day } & C & 36.2 & 35.3 & 33.9 & 35.0 & 28.5 & 28.8 & 28.9 & 30.0 & 28.9 & 27.5 & $31.3 \pm 3.4$ \\
\hline & $d$ & 34.7 & 33.2 & 36.3 & 35.9 & 35.3 & 29.7 & 29.5 & 30.6 & 28.9 & 28.9 & $32.3 \pm 3.1$ \\
\hline & a & 35.7 & 37.7 & 34.9 & 34.7 & 34.1 & 28.9 & 29.0 & 28.0 & 31.0 & 28.4 & $32.2 \pm 3.6$ \\
\hline 2 & $b$ & 35.3 & 35.3 & 39.1 & 33.4 & 32.7 & 30.0 & 28.3 & 31.6 & 29.5 & 30.0 & $32.5 \pm 3.3$ \\
\hline \multirow[t]{3}{*}{ day } & C & 37.1 & 34.1 & 35.8 & 30.0 & 35.6 & 28.3 & 30.0 & 30.9 & 29.1 & 29.2 & $32.0 \pm 3.3$ \\
\hline & $d$ & 33.3 & 36.0 & 34.1 & 34.2 & 36.4 & 30.0 & 30.9 & 30.0 & 30.0 & 30.0 & $32.5 \pm 2.6$ \\
\hline & a & 36.1 & 36.1 & 35.0 & 36.9 & 38.0 & 32.3 & 30.0 & 31.1 & 30.9 & 29.2 & $33.6 \pm 3.2$ \\
\hline 3 & $b$ & 34.6 & 35.8 & 40.5 & 36.2 & 33.0 & 31.6 & 28.6 & 31.6 & 30.0 & 28.4 & $33.0 \pm 3.8$ \\
\hline \multirow[t]{3}{*}{ day } & c & 37.9 & 36.9 & 35.4 & 36.4 & 31.4 & 30.1 & 30.0 & 30.0 & 30.3 & 30.0 & $32.8 \pm 3.4$ \\
\hline & $d$ & 36.3 & 37.6 & 35.4 & 38.9 & 35.1 & 27.3 & 31.5 & 29.4 & 29.4 & 30.7 & $33.2 \pm 4.0$ \\
\hline & a & 37.3 & 38.7 & 36.4 & 35.4 & 36.4 & 32.3 & 31.3 & 30.0 & 32.7 & 30.0 & $34.1 \pm 3.2$ \\
\hline 4 & $b$ & 35.3 & 36.2 & 33.4 & 41.2 & 36.7 & 28.3 & 28.9 & 30.0 & 31.6 & 32.1 & $33.4 \pm 4.0$ \\
\hline \multirow[t]{3}{*}{ day } & C & 38.3 & 36.7 & 37.1 & 32.7 & 37.1 & 29.4 & 30.0 & 30.0 & 30.3 & 31.3 & $33.3 \pm 3.6$ \\
\hline & $d$ & 38.4 & 35.7 & 36.7 & 40.0 & 36.7 & 30.8 & 30.0 & 30.1 & 29.4 & 32.6 & $34.0 \pm 3.9$ \\
\hline & a & 37.4 & 36.3 & 37.2 & 38.8 & 35.5 & 32.0 & 30.6 & 33.4 & 30.6 & 31.1 & $34.3 \pm 3.1$ \\
\hline 5 & $b$ & 37.1 & 37.7 & 35.9 & 41.6 & 33.8 & 29.0 & 32.0 & 30.0 & 29.0 & 32.6 & $33.9 \pm 4.2$ \\
\hline \multirow[t]{3}{*}{ day } & c & 39.0 & 37.4 & 37.4 & 33.1 & 38.2 & 30.1 & 30.5 & 29.1 & 29.4 & 30.0 & $33.4 \pm 4.1$ \\
\hline & $d$ & 38.2 & 38.6 & 40.2 & 36.1 & 37.1 & 30.7 & 33.2 & 30.1 & 31.3 & 30.0 & $34.6 \pm 3.9$ \\
\hline & a & 38.0 & 38.5 & 37.6 & 39.3 & 35.8 & 30.8 & 31.9 & 31.3 & 31.1 & 33.5 & $34.8 \pm 3.4$ \\
\hline 6 & $b$ & 38.1 & 42.2 & 36.9 & 37.6 & 33.4 & 30.7 & 30.0 & 29.3 & 33.7 & 31.8 & $34.3 \pm 4.2$ \\
\hline \multirow[t]{3}{*}{ day } & C & 40.1 & 38.2 & 37.7 & 38.7 & 34.0 & 30.1 & 31.0 & 30.5 & 31.9 & 30.7 & $34.3 \pm 4.0$ \\
\hline & $d$ & 37.3 & 39.1 & 41.3 & 37.4 & 38.3 & 30.0 & 33.7 & 30.5 & 31.7 & 31.4 & $34.1 \pm 4.1$ \\
\hline & a & 38.6 & 36.7 & 40.0 & 38.0 & 40.6 & 31.8 & 31.1 & 31.2 & 32.6 & 34.5 & $35.5 \pm 3.7$ \\
\hline 7 & b & 38.7 & 35.1 & 42.8 & 37.5 & 39.2 & 32.3 & 32.2 & 31.5 & 29.4 & 32.9 & $35.2 \pm 4.2$ \\
\hline \multirow[t]{2}{*}{ day } & C & 35.4 & 38.7 & 41.4 & 38.9 & 38.9 & 32.0 & 31.3 & 31.3 & 31.5 & 31.9 & $35.1 \pm 4.0$ \\
\hline & $d$ & 39.5 & 38.4 & 37.4 & 39.6 & 42.1 & 30.0 & 31.0 & 30.0 & 31.3 & 32.8 & $35.2 \pm 4.6$ \\
\hline
\end{tabular}

